\title{
Title: Functional coupling between target selection and acquisition in the superior colliculus
}

\author{
Jaclyn Essig and Gidon Felsen*
}

Department of Physiology and Biophysics, and Neuroscience Program

University of Colorado School of Medicine, Aurora, CO 80045, United States of America

Corresponding author: Gidon Felsen

E-mail: gidon.felsen@cuanschutz.edu

\section{Conflict of interest}

The authors declare no competing financial interests.

\section{Acknowledgements}

This work was supported by the National Institutes of Health (R01NS079518, F31NS103305), Rocky Mountain Neurological Disorders Center (P30NS048154), by $\mathrm{NIH/NCRR} \mathrm{Colorado} \mathrm{CTSI} \mathrm{grant} \mathrm{UL1} \mathrm{RR025780,} \mathrm{and} \mathrm{by} \mathrm{the} \mathrm{University} \mathrm{of} \mathrm{Colorado}$ NeuroTechnology Center. We thank Taylor Yamauchi and other members of the Felsen lab for their comments on the manuscript and Nathan D. Baker for technical assistance. Light microscopy was performed at the University of Colorado Anschutz Medical Campus Advance Light Microscopy Core and engineering support was provided by the University of Colorado Optogenetics and Neural Engineering Core. 


\section{Abstract}

2 To survive in unpredictable environments, animals must continuously evaluate their surroundings for behavioral targets, such as food and shelter, and direct their movements to acquire those targets. Although the ability to accurately select and acquire spatial targets depends on a shared network of brain regions, how these processes are linked by neural circuits remains unknown. The superior colliculus (SC) mediates the selection of spatial targets and remains active during orienting movements to acquire targets, which suggests the underexamined possibility that common SC circuits underlie both selection and acquisition processes. Here, we test the hypothesis that SC functional circuitry couples target selection and acquisition using a default motor plan generated by selection-related neuronal activity. Single-unit recordings from intermediate and deep layer SC neurons in male mice performing a spatial choice task demonstrated that choice-predictive neurons, including optogenetically identified GABAergic SC neurons whose activity was causally related to target selection, exhibit increased activity during movement to the target. By strategically recording from both rostral and caudal SC neurons, we also revealed an overall caudal-to-rostral shift in activity as targets were acquired. Finally, we used an attractor model to examine how target selection activity in the SC could generate a rostral shift in activity during target acquisition using only intrinsic SC circuitry. Overall, our results suggest a functional coupling between SC circuits that underlie target selection and acquisition, elucidating a key mechanism for goal-directed behavior.

\section{Significance Statement}

22 The ability to quickly select and acquire spatial targets is essential to animal survival. Neural

23 circuits underlying these processes are shared in an interconnected network of brain regions, however it is unclear how circuits link decision-making processes with motor commands to execute choices. Here, we examine single-unit activity in the superior 
colliculus (SC) as mice select and acquire spatial targets to test the hypothesis that choice-

related activity promotes target acquisition by generating a default motor plan for orienting

movements. By demonstrating that choice-predictive neurons increase their firing rates

during movement and capturing the dynamics of SC activity with an attractor model of intrinsic SC circuitry, our results support a role for SC circuits in coupling target selection

31 and acquisition.

\section{Introduction}

Animals continuously evaluate and approach spatial goals while interacting with their environment (Cisek and Kalaska, 2010). Effective goal-directed behavior requires the coordination of sensory, cognitive, and motor processes to accurately identify, select, and acquire spatial targets such as food or mates. Despite being traditionally conceptualized and examined as independent sequential processes whereby decisions are deliberated before actions are planned (Miller et al., 1960), target selection and acquisition in natural environments are fluid processes that continuously influence each other (Cisek and PastorBernier, 2014). However, little is known about how the neural circuits underlying target selection and acquisition are integrated to coordinate goal-directed behavior.

A key node in the network of brain regions mediating goal-directed behaviors is the superior colliculus (SC), an evolutionarily conserved midbrain structure with sensorimotor properties well suited to support target selection and acquisition (Krauzlis et al., 2004; Stein and Stanford, 2008; Gandhi and Katnani, 2011; Wolf et al., 2015; Basso and May, 2017). rostrocaudal axis such that targets eccentric from the midline are encoded caudally and, as the target is acquired, activity emerges rostrally (Munoz et al., 1991; Anderson et al., 1998; Port et al., 2000; Choi and Guitton, 2009; Gandhi and Katnani, 2011). In general, rostral 
51 and, in the case of eye movements, microsaccades and fixation (Munoz and Wurtz, 1993;

52 Munoz and Istvan, 1998; Basso et al., 2000; Krauzlis, 2003; Choi and Guitton, 2006; Goffart et al., 2012). Extensive sets of studies have focused on the role of the SC in both target selection (Sparks and Mays, 1980; Horwitz and Newsome, 1999; Glimcher, 2001; McPeek and Keller, 2002; Krauzlis et al., 2004; Felsen and Mainen, 2008; Duan et al., 2015; Basso and May, 2017) and acquisition (Guitton et al., 2003; Gandhi and Katnani, 2011; Marino et al., 2012; Smalianchuk et al., 2018), but it is unclear how these processes are related at the level of SC circuits. One possibility is that distinct sources of cortical and subcortical input to specific cell types along the rostrocaudal axis (Benavidez et al., 2020; Doykos et al., 2020) can mediate selection and acquisition via independent collicular pathways. Another underexamined possibility is that target selection and acquisition are intrinsically linked via SC circuitry. Given the tight linkage between these processes - acquisition must often follow soon after selection - shared neural circuitry could provide behavioral and computational advantages (Wolpert and Landy, 2012; Cisek, 2019). Although much remains unknown about the functional organization of SC circuits, several recent studies have characterized the roles of specific SC cell types in behavior (Masullo et al., 2019; Essig et al., 2020; Wang et al., 2020) and the sophistication of SC circuitry has been increasingly appreciated.

Here, we test the hypothesis that SC circuits are configured such that neural activity underlying the selection of a target promotes a default motor plan for acquiring the target via an orienting movement. Under this hypothesis, we would expect that SC activity causal for target selection would initialize activity for target acquisition which may subsequently be modulated by external input. We recorded rostral and caudal SC activity, including from optogenetically-identified GABAergic neurons, in male mice selecting and acquiring spatial 
rates during target acquisition, consistent with previous work (Munoz and Wurtz, 1995b;

Bergeron et al., 2003; Choi and Guitton, 2009), while many caudal SC neurons exhibited activity related to target selection and remained active during target acquisition. rostrocaudal location. We next developed an attractor model to examine how intrinsic SC circuitry could promote a shift of activity from caudal to rostral SC during target acquisition. Overall, our findings suggest that SC circuitry may facilitate goal-directed behavior by coupling target selection and acquisition.

Materials and Methods

Data were collected during experiments previously described in a published study focusing on spatial choice (Essig et al., 2020). All analyses and results presented in the current study are novel. All methodology relevant to the current study is provided here.

\section{Experimental Design}

\section{Animals}

All procedures were approved by University of Colorado School of Medicine Institutional Animal Care and Use Committee. Mice were bred in the animal facilities of the University of Colorado Anschutz Medical Campus. Heterozygous Gad2-ires-Cre (Gad2-Cre; $\left.\operatorname{Gad2}^{\mathrm{tm} 2(\mathrm{cre}) \mathrm{Zjh} / \mathrm{J})}\right)$ male mice $(\mathrm{n}=10 ; 6-12$ months old at time of experiments) were used in this study. Mice were housed individually in an environmentally controlled room, kept on a 12-hour light/dark cycle and had ad libitum access to food. Mice were water restricted to 1 $\mathrm{ml} /$ day and maintained at $80 \%$ of their adult weight.

\section{Behavioral task}

98 Mice were trained on a previously published odor-guided spatial-choice task (Uchida and Mainen, 2003; Stubblefield et al., 2013; Essig et al., 2020). Briefly, water-restricted mice self-initiated each trial by nose poking into a central port. After a short delay ( 200 ms), a 
101 binary odor mixture was delivered. Mice were required to wait $500 \pm 55 \mathrm{~ms}$ (mean $\pm \mathrm{SD}$ ) for

102 a go signal (a high frequency tone) before exiting the odor port and orienting toward the left 103 or right reward port for water (Fig. 1A). We refer to the time between odor valve open and

104 the go signal as the "choice epoch" (Figs. 1D,E; 3). Exiting the odor port prior to the go

105 signal resulted in the unavailability of reward on that trial, although we still analyzed these

106 trials if a reward port was selected. All training and experimental behavioral sessions were 107 conducted during the light cycle.

(Odor B) (Acros), commonly perceived as caraway and spearmint, respectively. In all

sessions - including training on the task, as well as during neural recording and

manipulation - mixtures in which Odor A > Odor B indicated reward availability at the left

112 port, and Odor $B$ > Odor $A$ indicated reward availability at the right port (Fig. 1A). When

113 Odor $\mathrm{A}=$ Odor $\mathrm{B}$, the probability of reward at the left and right ports, independently, was

114 0.5. The full set of Odor A/Odor B mixtures used was 95/5, 80/20, 60/40, 50/50, 40/60,

$11520 / 80,5 / 95$ and mouse performance on the task was dependent on odor concentration (see

116 Essig et al., 2020). Mice completed training in 8-12 weeks and were then implanted with a

117 neural recording drive. All neural recordings were performed in mice that were well-trained

118 on the task.

\section{Stereotactic surgeries}

120 Mice were removed from water restriction and had ad libitum access to water for at least

121 one week before surgery. Preparation for surgery was similar for viral injections and chronic

122 implants. Deep anesthesia was induced with $2 \%$ isoflurane (Priamal Healthcare Limited) in

123 a ventilated chamber before being transferred to a stereotaxic frame fitted with a heating

124 pad to maintain body temperature. A nose cone attachment continuously delivered $1.3 \%-$

$1251.6 \%$ isoflurane to maintain anesthesia throughout the surgery. Scalp fur was removed 
using an electric razor and ophthalmic ointment was applied to the eyes. The scalp was cleaned with betadine (Purdue Products) and 70\% ethanol before injecting a bolus of topical anesthetic (150 $\mu \mathrm{l} 2 \%$ lidocaine; Aspen Veterinary Resources) under the scalp. The skull was exposed with a single incision and scalp retraction. The surface of the skull was cleaned with saline and the head was adjusted to ensure lambda was level with bregma (within $150 \mu \mathrm{m}$ ). Immediately following all surgeries, mice were intraperitoneally administered sterile $0.9 \%$ saline for rehydration and an analgesic ( $5 \mathrm{mg} / \mathrm{kg} \mathrm{Ketofen;} \mathrm{Zoetis).}$ A topical antibiotic was applied to the site of incision and mice were given oxygen while waking from anesthesia. Post-operative care, including analgesic and antibiotic administration, continued for up to 5 days after surgery and mice were closely monitored for signs of distress. Additionally, mice recovered after surgery with ad libitum access to water for at least 1 week before being water-restricted for experiments.

For viral injections, a small craniotomy was drilled above the left SC at rostral (3.16$3.4 \mathrm{~mm}$ posterior to bregma, $0.75-1 \mathrm{~mm}$ lateral of midline, $1.8-2.17 \mathrm{~mm}$ dorsal from the brain surface; $\mathrm{n}=3$ mice) (Isa et al., 2020) or caudal (3.5 - 4 mm posterior to bregma, 0.8 $1.25 \mathrm{~mm}$ lateral of midline, $0.85-1.5 \mathrm{~mm}$ dorsal from the brain surface; $\mathrm{n}=7$ mice) locations (Franklin and Paxinos, 2008). To maximize overlap between ChR2 expression and the optetrode in Gad2-Cre mice, up to 3 injections were made within $0.2 \mathrm{~mm}^{2}$. Viruses were delivered with a thin glass pipette at an approximate rate of $100-200 \mathrm{nl} / \mathrm{min}$ via manual pressure applied to a $30 \mathrm{ml}$ syringe. Pipets remained at depth for $10 \mathrm{~min}$ following each injection before retraction from the brain. Mice were injected with a total (across all injections) of $200 \mathrm{nl}$ of DIO-ChR2-eYFP (AAV2.Ef1a.DIO.ChR2.eYFP, UNC Vector Core, $\left.4.2 \times 10^{12} \mathrm{ppm}\right)$. After injection, the skin was sutured and mice recovered for 1 week before being water restricted for behavioral training. Expression occurred during the $\sim 10$ weeks of training. 
To extracellularly record from optogenetically-identified GABAergic SC neurons, an

152 optetrode drive, an optic fiber surrounded by four tetrodes (Anikeeva et al., 2012), was

153 chronically implanted above the DIO-ChR2-eYFP injection site in fully-trained Gad2-Cre

154 mice. A large $\left(\sim 1 \mathrm{~mm}^{2}\right)$ craniotomy was made around the initial injection site. Three

155 additional small craniotomies were made anterior to the initial injection site: one for

156 implanting a ground wire and two for skull screws. The drive was slowly lowered into the

157 large craniotomy and secured in place with luting and dental acrylic.

\section{Electrophysiology}

159 Extracellular neuronal recordings were collected using four tetrodes (a single tetrode

160 consisted of four polyimide-coated nichrome wires (Sandvik; single-wire diameter $12.5 \mu \mathrm{m}$ )

161 gold plated to $0.25-0.3 \mathrm{M} \Omega$ impedance). Electrical signals were amplified and recorded

162 using the Digital Lynx S multichannel acquisition system (Neuralynx) in conjunction with

163 Cheetah data acquisition software (Neuralynx).

To sample independent populations of neurons, the tetrodes were advanced

between 6 - $23 \mathrm{~h}$ before each recording session. To estimate tetrode depths during each

session we calculated distance traveled with respect to rotation fraction of the thumb screw

167 of the optetrode drive. One full rotation moved the tetrodes $\sim 450 \mu \mathrm{m}$ and tetrodes were

168 moved $\sim 100 \mu \mathrm{m}$ between sessions. The final tetrode location was confirmed through

169 histological assessment.

171 software (MClust 4.4.07, A.D. Redish) in MATLAB (2015a). Briefly, for each tetrode, single

172 units were isolated by manual cluster identification based on spike features derived from

173 sampled waveforms. Identification of single units through examination of spikes in high-

174 dimensional feature space allowed us to refine the delimitation of identified clusters by

175 examining all possible two-dimensional combinations of selected spike features. We used 
standard spike features for single unit extraction: peak amplitude, energy (square root of the sum of squares of each point in the waveform, divided by the number of samples in the waveform), valley amplitude and time. Spike features were derived separately for individual leads. To assess the quality of identified clusters we calculated isolation distance, a standard quantitative metric (Schmitzer-Torbert et al., 2005). Clusters with an isolation distance $>6$ were deemed single units. Units were clustered blind to interspike interval, and only clusters with few interspike intervals $<1 \mathrm{~ms}$ were considered for further examination. Furthermore, we excluded the possibility of double counting neurons by ensuring that the waveforms and response properties sufficiently changed across sessions. If they did not, we conservatively assumed that we recorded twice from the same neuron, and only included data from one session.

Electrophysiological recordings were obtained from 285 SC neurons in 96 behavioral sessions. Details of our analyses of the data obtained from our recording experiments are described below. All neural data analyses were performed in MATLAB (2015a/2019a). Neurons recorded during behavior/recording sessions with fewer than 40 trials in either direction or with a choice-epoch and movement-epoch firing rate below 2.5 spikes/s for trials in both directions were excluded from all analyses, resulting in 285 neurons in this data set.

\section{Histology}

Final tetrode location was confirmed histologically (see Essig et al., 2020). Mice were overdosed with an intraperitoneal injection of sodium pentobarbital (100 mg/kg; Sigma Life Science) and transcardially perfused with phosphate buffered saline (PBS) and 4\% paraformaldehyde (PFA) in $0.1 \mathrm{M}$ phosphate buffer (PB). After perfusion, brains were submerged in 4\% PFA in $0.1 \mathrm{M} \mathrm{PB}$ for $24 \mathrm{hr}$ for post-fixation and then cryoprotected for at least $12 \mathrm{hr}$ immersion in $30 \%$ sucrose in $0.1 \mathrm{M} \mathrm{PB}$. On a freezing microtome, the brain was 
201 frozen rapidly with dry ice and embedded in 30\% sucrose. Serial coronal sections (50 $\mu \mathrm{m})$

202 were cut and stored in 0.1M PBS. Sections were stained with 435/455 blue fluorescent Nissl

203 (1:200, NeuroTrace; Invitrogen) to identify cytoarchitectural features of the SC and verify

204 tetrode tracks to determine the depth and rostrocaudal location of the recordings.

205 Optogenetic identification of GABAergic neurons

206 Before and/or after behavioral sessions, light was delivered via a diode-pumped, solid-state 207 laser (473 nm; Shanghai Laser \& Optics Century) at $8 \mathrm{~Hz}$ (10 ms on) or, for a small sample

208 (<20 sessions), at $25 \mathrm{~Hz}$ (10 ms on) to record extracellular light-induced activity from the

209 same population of neurons that were recorded during behavior. Isolated units were

210 identified as GABAergic based on reliable, short-latency responses to light and high

211 waveform correlations between spontaneous and light-evoked action potentials (see Essig

212 et al., 2020 for further details). Neurons identified as GABAergic could be further identified

213 (i.e., "tracked") during behavioral sessions based on spike features and location (i.e.,

214 tetrode number and lead number).

\section{Choice prediction}

216 To examine the dependence of the firing rate of individual neurons on choice (Fig. 1C-E),

217 we used an ROC-based analysis (Green and Swets, 1966) that quantifies the ability of an

218 ideal observer to classify whether a given spike rate during the choice epoch was recorded

219 in one of two conditions (here, during ipsilateral or contralateral trials)(Feierstein et al.,

220 2006; Essig et al., 2020). We defined the choice epoch as beginning $100 \mathrm{~ms}$ after odor

221 valve open and ending with the go signal. Statistical significance was determined with a

222 permutation test: we recalculated choice prediction (CP) after randomly reassigning all firing

223 rates to either of the two groups arbitrarily, repeated this procedure 500 times to obtain a

224 distribution of values, and calculated the fraction of random values exceeding the actual

225 value. We tested for significance at $\alpha=0.05$. Using this analysis we found that $55 / 285$ 
226

227

228

229

230

231

232

233

234

235

236

237

238

239

240

241

242

243

244

245

246

247

248

249

neurons predicted contralateral choice $(\mathrm{CP}>0, p<0.05)$ and 30/285 neurons predicted ipsilateral choice (CP < 0, $p<0.05)$ (Fig. 1C-E); see Essig et al. (2020) for more details).

\section{Comparing activity during choice and movement epochs}

To examine activity across the choice and movement epochs of choice-predictive neurons $(p<0.05)$, activity was averaged across contralateral and ipsilateral trials (separately) in $10 \mathrm{~ms}$ bins (Fig. 1C). The activity of each neuron was rescaled across both directions and epochs from 0 (lowest activity; black) to 1 (highest activity; white), smoothed with a gaussian filter $(\sigma=40 \mathrm{~ms})$ and sorted by timing of maximum firing rate. Example peristimulus time histograms in Figure 1B were smoothed with a Gaussian filter $(\sigma=10$ $\mathrm{ms})$.

We compared median population firing rates for all choice-predictive neurons and GABAergic choice-predictive neurons between the choice epoch (100 ms after odor delivery to go signal; 400 ms) and movement epoch (center port exit to target port; 630 ms) separately for contralateral choice-predictive and ipsilateral choice-predictive neurons (Fig. 1D,E). Neurons were included for analysis if their mean firing rate across the sessions was $>1 \mathrm{spk} / \mathrm{s}$ for at least 1 epoch (choice or movement) in the direction analyzed (ipsilateral or contralateral trials). Mann-Whitney U tests were performed for all comparisons.

To examine firing rate throughout the movement epoch, activity was averaged across contralateral and ipsilateral trials (separately) in $10 \mathrm{~ms}$ bins (Fig. 2A). The activity of each neuron was rescaled across both directions and both epochs from 0 (lowest activity; black) to 1 (highest activity; white), smoothed with a gaussian filter ( $\sigma=20 \mathrm{~ms}$ ) and sorted by the time of maximum firing rate. Neurons with peak firing rates at the time of target entry were omitted from analysis ( $\mathrm{n}=42$ neurons).

\section{Attractor model}


250

251

252

253

254

255

256

257

258

259

260

261

262

263

264

265

266

267

268

269

270

$271[2]$

272 by

We adapted a rate-based bump attractor model originally developed to study spatial choice (Lintz et al., 2019; Essig et al., 2020) to examine rostrocaudal activity dynamics during movement. As detailed in Essig et al. (2020) and briefly described here, the model consists of 200 excitatory $(E)$ cells and 100 inhibitory $(I)$ cells per SC (600 cells total). Intra-SC synaptic weights were larger for nearby cells, and smaller for more distant ones, determined

$$
\text { [1] } \quad W_{i j}=a m p * e^{-(i-j)^{2} / s c a l e^{2}},
$$

where $i$ and $j$ are the locations of the pre- and post-synaptic cells, respectively, and amp and scale are defined independently for presynaptic $E$ and / cells (ampE $=0.01$, $a m p l=0.15$, scaleE $=0.03$ ). amp sets the amplitude (i.e., "strength") of the connection weights, and scale determines the spatial extent over which the connection strength decays. Overall, I cells had a higher scale than E cells, with scalel magnitude determined by the rostral ( / cells 1:25) or caudal ( / cells $26: 100)$ location of the presynaptic cell: scalel rostral $=0.5$ and scalel $_{\text {caudal }}=0.4$. Inter-SC synaptic connections were made sparse by assigning synaptic weights to a subset of probabilistically determined contralateral $E$ and I cells and were reciprocated between the left and right SC. To promote network stability, each $W$ was normalized to have a maximum eigenvalue of 1.5 by dividing all connection values by $\max (\lambda) / 1.5$, where $\max (\lambda)$ is the largest eigenvalue of the matrix after initialization. Spike rates of $E$ and I cells in the left SC ( $r_{E}^{L}$ and $r_{I}^{L}$, respectively) evolved at each time step ( 2 ms in our numerical simulations) according to

$$
\tau \frac{d v_{E}^{L}}{d t}=-v_{E}^{L}+W_{E E}^{L} r_{E}^{L}-W_{I E}^{L} r_{I}^{L}+W_{R_{E} \rightarrow L_{E}}^{L} r_{E}^{R}-W_{R_{I} \rightarrow L_{E}}^{L} r_{I}^{R}+\text { external } L^{L}+\text { noise }
$$

$$
\tau \frac{d v_{I}^{L}}{d t}=-v_{I}^{L}-W_{I I}^{L} r_{I}^{L}+W_{E I}^{L} r_{E}^{L}-W_{R_{I} \rightarrow L_{I}}^{L} r_{I}^{R}+W_{R_{E} \rightarrow L_{I}}^{L} r_{E}^{R}+\text { external }^{L}+\text { noise }
$$


273 and all spike rates were rectified at each time step according to

274 [3] $\quad r=\left\{\begin{array}{lr}0, & \text { for } v<0 \\ V, & \text { for } 0<v<100 \\ 100, & \text { for } v>100\end{array}\right.$

275 where, e.g., $W_{E E}^{L}$ represents synaptic weights from left $E$ to $E$ cells, $W_{I E}^{L}$ represents weights

276 from left / to $E$ cells, and $W_{R_{I} \rightarrow L_{I}}^{L}$ represents weights from right / to left $E$ cells. Noise was

277 drawn from a Gaussian distribution with mean $=0$, and variance $=15$ for $E$ cells and 5 for $I$

278 cells. $r_{E}^{L}$ and $r_{I}^{L}$ are vectors, with one entry per $E$ or I cell in the left SC, respectively.

279 Similarly, $r_{E}^{R}$ and $r_{I}^{R}$ describe the firing rates of cells in the right SC, and they evolve over

280 time via the same equations as those in the left SC (i.e., via Eqs. [2], [3], with all "L"S and

281 "R"s swapped).

The vectors external ${ }^{L}$ and external ${ }^{R}$ represent the drive to the $E$ and I cells from

sources outside the SC. For all trial types, external drive was applied in a linearly graded fashion to a small fraction of the most caudal cells ( $E$ cells numbered 170 to 179 and $/$ cells numbered 85 to 89 ). On leftward trials, the right SC received a stronger external drive than the left SC, and vice versa on rightward trials. For each trial, external drive began at time step 151, corresponding to the odor delivery time in the task, and stopped time step 400 for an approximate total time of $\sim 500 \mathrm{~ms}$. At step 401, immediately following removal of the external input, choice was determined based on the SC with the highest average caudal $E$ cell firing rate. Each "session" consisted of 255 trials, with the proportion of different

291 difficulties matched to the behavioral sessions. Each model data point consists of 20 sessions (Fig. 4C,D; 5A,C). 


\section{Statistical Analysis}

297 In general, normality of distributions was tested using the Anderson-Darling test. Data were analyzed using MATLAB (2015a/2019a) and presented primarily as medians or means \pm SEM. $p$ values for each comparison are reported in the figure legends, results and/or methods sections. Mice were excluded from analyses based on the misalignment of the optetrode with ChR2 expression, lack of ChR2 expression, and misplacement of the optetrode outside of the SC (13 mice were excluded from the final data set).

Code Accessibility

304 The data that support the findings of this study and the custom MATLAB code are available

305 from the corresponding author upon written request.

\section{Results}

Activity in choice-predictive SC neurons increases during movement to selected targets

To begin testing the hypothesis that selecting a target promotes a default motor plan in the superior colliculus (SC) for acquiring the target, we examined how neural activity evolved in the SC of freely-moving mice selecting and acquiring targets in a two-alternative spatial

311 choice task (Stubblefield et al., 2013; Essig et al., 2020). The task required mice to first

312 enter a central port and to sample a binary mixture of odors. Mice were trained to select 313 either the left or right reward port based on the dominant component of the odor mixture

314 ("Choice"; Fig. 1A) and, at the presentation of a go signal, initiate an orienting movement 315 from the center port to the selected reward port to retrieve a water reward ("Movement"; Fig.

316 1A). As mice performed the task, we obtained single-unit recordings, including from

317 identified GABAergic neurons (Materials and Methods; see Essig et al., 2020), from the 318 intermediate and deep layers of the SC. The dissociation of choice and movement epochs enabled us to compare choice- and movement-related activity in our recorded population.

320 Consistent with previous reports, including of primates performing saccadic eye movements 
321 (Munoz and Wurtz, 1995a; Basso and Wurtz, 1998; Everling et al., 1999; Felsen and

322 Mainen, 2008), many neurons displayed activity throughout choice and movement (Fig. 1B).

324 that likely causally contribute to target selection (Essig et al., 2020). Briefly, neurons are

325 classified as choice predictive based on an ROC analysis of their firing rates during the

326 choice epoch (Materials and Methods). For example, neurons that exhibit significantly

327 higher firing rate when selecting the contralateral than the ipsilateral target would be classified as contralateral choice-predictive neurons. We found that choice-predictive neurons ( $n=85 / 285$ neurons, $p<0.05$ ) appeared to be highly active during movement (Fig.

1C). We quantified the change in activity of these neurons between the choice epoch

331 (during odor sampling; $400 \mathrm{~ms}$ ) and the movement epoch (from center port to reward port; $\sim 630 \mathrm{~ms}$ ). Since the activity of many SC neurons depends on movement direction (Horwitz and Newsome, 2001; Felsen and Mainen, 2008; Essig et al., 2020), we examined activity

334 for ipsilateral and contralateral trials separately. We first compared the firing rates for the choice epoch to the movement epoch on contralateral trials and found that for our total population of contralateral choice-predictive neurons, as well as for contralateral choice-

337 predictive neurons identified as GABAergic, activity significantly increased from choicerelated levels during movement ( $p<0.0001$, Wilcoxon signed-rank test; Fig. 1D). We did not find a similar increase in activity during the movement epoch for ipsilateral choice-

340 predictive neurons on ipsilateral trials ( $p>0.05$, Wilcoxon signed-rank test; Fig. 1E). These

341 results demonstrate that SC neurons mediating target selection are more active during 342 movement than during choice, even in identified GABAergic populations, consistent with the 343 hypothesis that choice-related activity is linked to a motor plan for target acquisition in the SC. 
$\mathrm{A} \leftarrow[\mathrm{A}]>[\mathrm{B}] \quad[\mathrm{B}]>[\mathrm{A}] \longrightarrow$
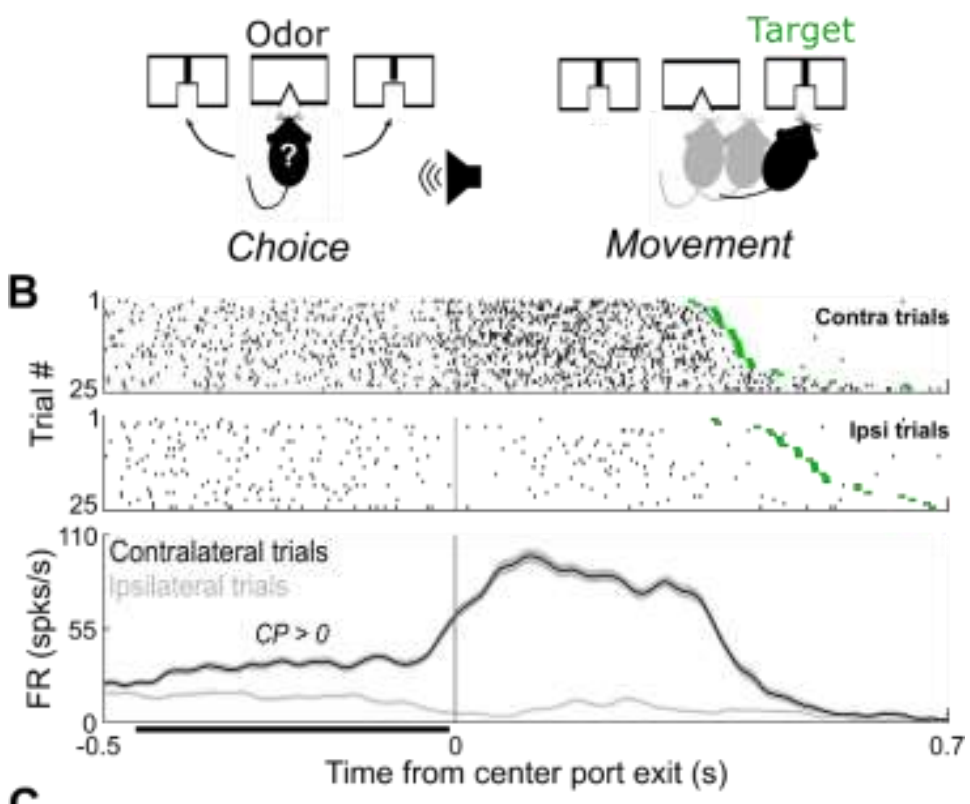

C
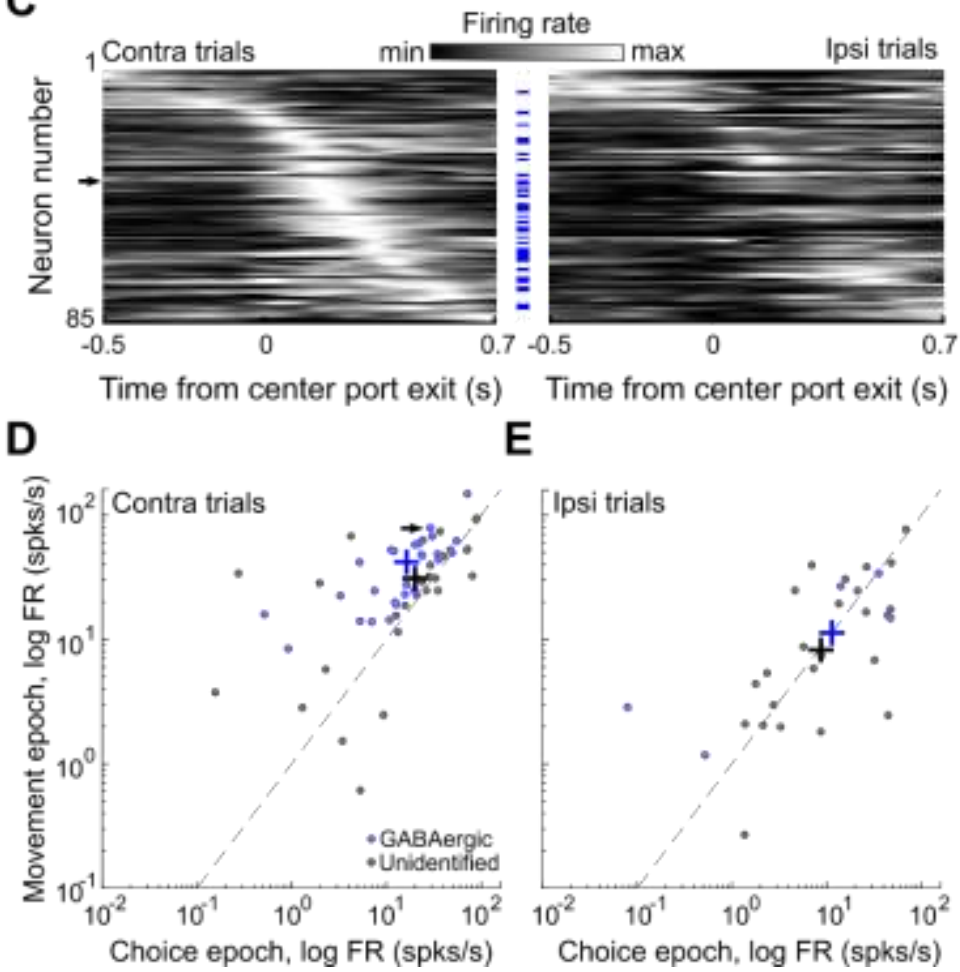

Figure 1: Choice-predictive SC neurons remain active during movement.

(A) Schematic of task. Mice choose the left or right target (reward port) based on an odor mixture ("Choice"). After a go signal, mice move to the chosen target for a water reward ("Movement"). (B) Rasters (top) and peri-event histograms (bottom) for a GABAergic SC neuron that predicts contralateral choice. Black bar indicates the "choice epoch" when the choice prediction of each neuron is calculated based on the area under the ROC curve constructed from the distributions of firing rates on ipsilateral and contralateral trials.(C) Normalized activity during choice and movement of choice-predictive SC neurons ( $n=85)$, 
separately for trials in which the contralateral and ipsilateral target was selected. Neurons are sorted by time of maximum firing rate. Blue dashes indicate GABAergic neurons. Arrow, example neuron in B. (D) Mean firing rates during the choice epoch plotted against mean firing rates during the movement epoch for each neuron that predicted contralateral choice $(n=55)$. Only trials in which the contralateral target was selected are shown. Contralateral choice-predictive neurons, including GABAergic neurons, exhibited higher mean activity during movement than choice $(p<0.0001$, Wilcoxon signed-rank tests). Crosshairs indicate population medians for all (black) and GABAergic (blue) neurons. Arrow, example neuron in B. (E) As in $\mathbf{D}$, epoch for each neuron that predicted ipsilateral choice $(n=30)$. Only trials in which the ipsilateral target was selected are shown. Activity in this population did not differ between the choice and movement epochs ( $p>0.05$, Wilcoxon signed-rank test).

\section{Rostral shift of activity during movement}

Given the high activity during movement, even among neurons likely involved in target selection (Essig et al., 2020), we next examined how the activity of our entire population of SC neurons evolves as mice are orienting to, and acquiring, spatial targets. We found that the majority of neurons were active during movement and, across the population, peak activity was exhibited throughout the duration of the movement (Fig. 2A). Interestingly, population activity during contralateral and ipsilateral orienting movements was similar, although, again consistent with a prominent role for the SC in mediating movements to contralateral targets (Gandhi and Katnani, 2011), the maximum firing rate for most neurons occurred during contralateral trials ( $n=144 / 243$ neurons). The activity of GABAergic neurons (Fig. 2A, blue dashes) tended to peak later than the overall population (total population median: 250 ms before target entry; GABAergic population median: $190 \mathrm{~ms} ; p=$ 0.021, Mann-Whitney U test).

We next analyzed the timing of maximum firing rate based on the rostrocaudal location of each neuron as previous studies have postulated that activity shifts from caudal to rostral SC as targets are acquired (Munoz et al., 1991; Guitton et al., 2003). By targeting our recordings to the rostral or caudal SC in separate sets of mice, we recorded from 73 rostral neurons (25 GABAergic) and 170 caudal neurons (58 GABAergic). 
A

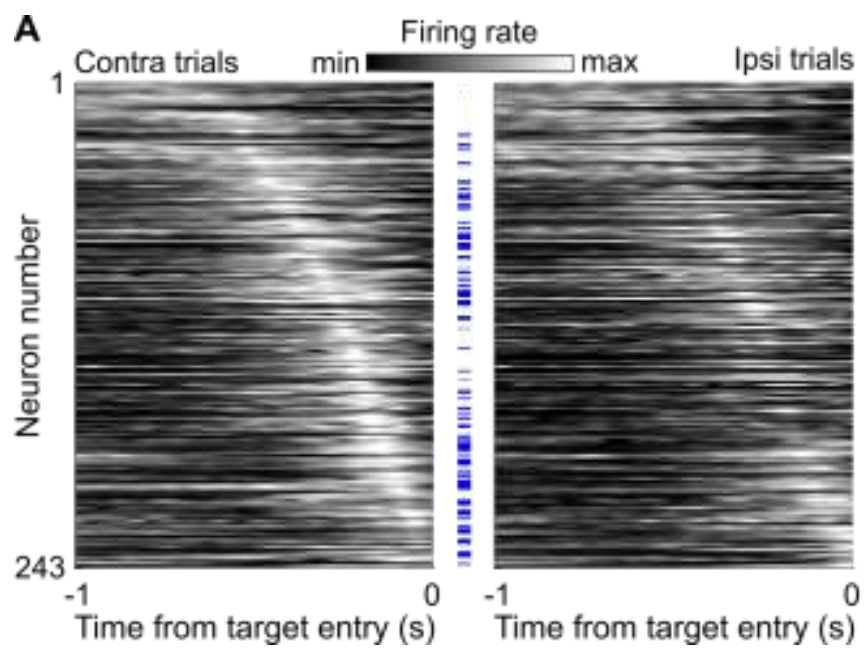

B Time of max firing rate

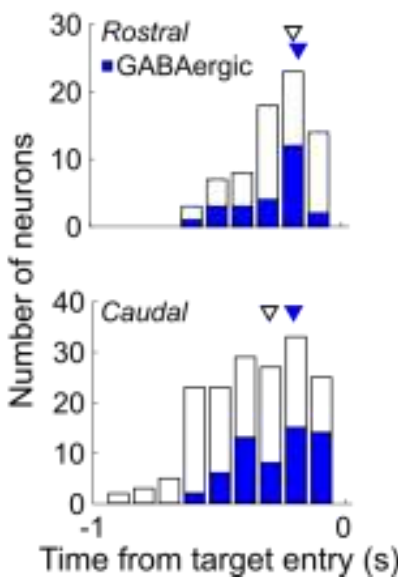

Figure 2: Timing of SC activity during movement to the target.

(A) Normalized activity of all SC neurons during movement, separately for trials in which the contralateral and ipsilateral target was selected. Neurons are sorted by time of maximum firing rate and omitted from analysis if their peak firing rate occurred at the time of target entry $(t=0 \mathrm{~s})$. Blue dashes indicate GABAergic neurons. Peak firing rate of GABAergic neurons occurs later than the overall population ( $p=0.021$, Mann-Whitney $U$ test). (B) Time of maximum firing rate separately for rostral and caudal populations. In the overall population, peak firing rate was later in rostral (top) than caudal neurons (bottom) ( $p=$ 0.00066, Mann-Whitney $U$ test). Arrowheads show median time of peak firing rate for overall (open) and GABAergic (blue) populations of neurons.

In the overall population, rostral neurons reached their maximum firing rates later than caudal neurons (Fig. 2B, white arrowheads; rostral: 190 ms before target entry; caudal: 245 $\mathrm{ms} ; p=0.00066$, Mann-Whitney $\mathrm{U}$ test). These results are consistent with $\mathrm{SC}$ activity recorded from cats and primates performing similar tasks (Munoz et al., 1991; Munoz and Wurtz, 1995b, 1995a) and suggest that, as a population, SC represents movements to spatial targets. Interestingly, the activity of GABAergic neurons did not depend on

404 rostrocaudal location and were active significantly later during movement than the total 405 population (Fig. 2B, blue arrowheads; rostral: 170 ms before target entry; caudal: 205 ms; $p$ $406=0.92$, Mann-Whitney $U$ test), consistent with a role for GABAergic neurons in target 407 acquisition, in addition to their role in target selection (Essig et al., 2020). 
Rostral SC activity increases as targets are acquired

409 To more rigorously examine differences in activity between rostral and caudal neurons, we

410 quantified their activity in epochs capturing key features of the task: choice, movement

411 initiation, target acquisition and odor port re-entry (Fig. 3A). As in Figure 1D and E, the

412 choice epoch was defined as when mice are in the center port and sampling the odor ( 400

413 ms; Fig. 3A). The remaining epochs were $25 \mathrm{~ms}$ each and yoked to specific task events:

414 "movement initiation" (the 25 ms following odor poke out), "acquisition" (the 25 ms

415 preceding port entry) and "odor port re-entry" (the 25 ms preceding odor port entry; Fig. 3A).

416 We focused on contralateral trials since these generally elicited higher activity than

417 ipsilateral trials (Figs. 1C; 2A). Firing rates for GABAergic neurons did not differ from that of

418 the overall population in any epoch ( $p>0.05$, Mann-Whitney $U$ tests); we thus performed

419 our inter-epoch comparisons on the overall population.

420

We first examined the activity of neurons recorded in the rostral SC (Fig. 3B).

421 Overall, we found that rostral activity, while exhibiting significant variability within epochs,

422 was modulated between epochs in the task (Fig. 3B; $p=0.00077$, Kruskal-Wallis test).

423 Population activity of rostral neurons remained unchanged from choice to movement

424 initiation $(p=0.66)$, however, there was a significant increase in activity during acquisition

425 compared to choice $(p=0.00041)$ and movement initiation ( $p=0.023$, multiple comparison

426 tests). Rostral activity during odor port re-entry did not differ from that during acquisition or

427 the other epochs, perhaps reflecting the fact that, within the context of the task, entering the

428 odor port could be considered a form of target acquisition, but was only indirectly linked to

429 the goal of obtaining water. Conversely, neurons recorded from the caudal SC maintained

430 choice-epoch firing rates for each subsequent epoch (Fig. $3 C ; p=0.46$, Kruskal-Wallis test). 

specifically as targets are acquired.
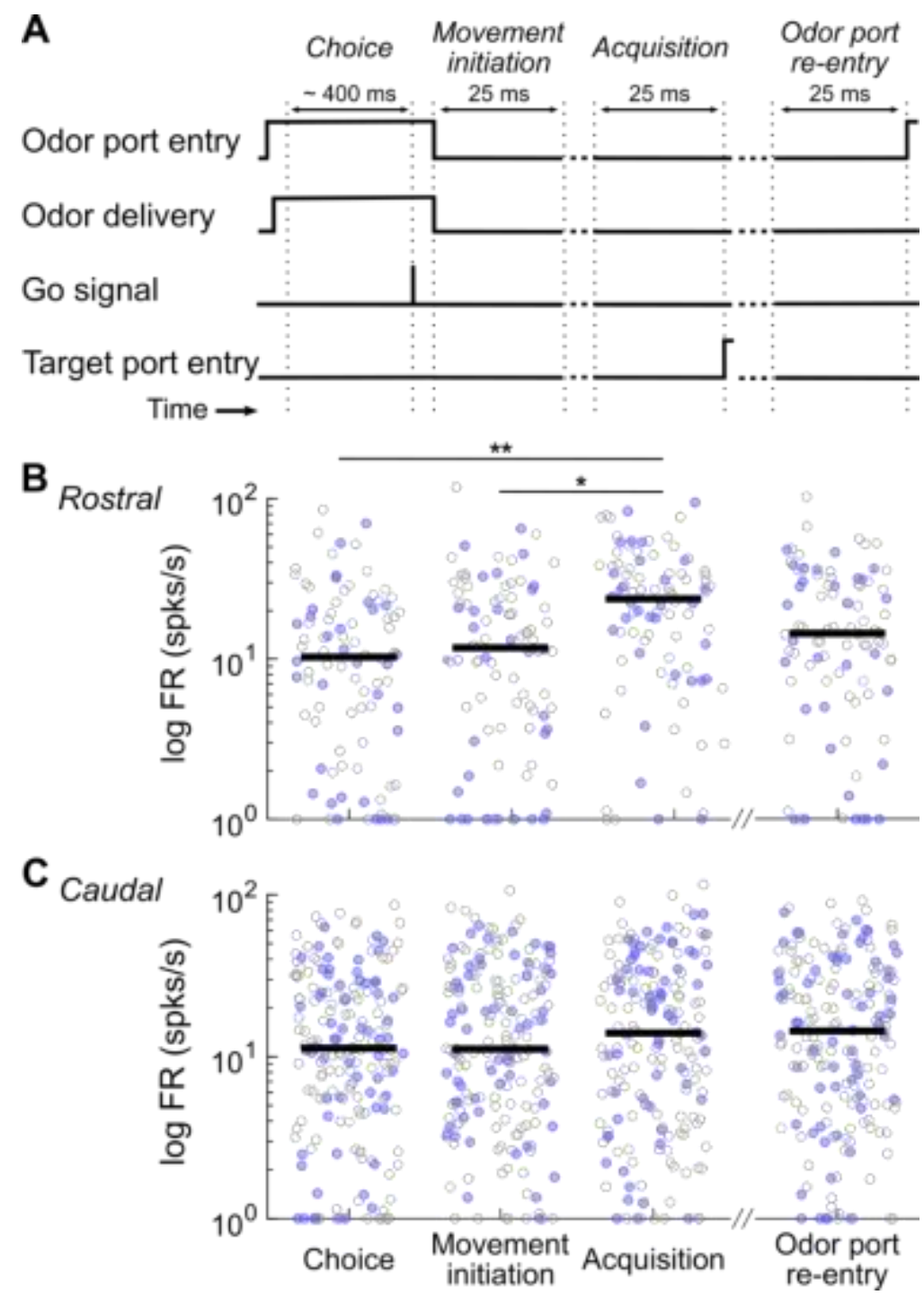

Figure 3: Comparison of SC activity across task epochs.

(A) Definitions of event-based epochs of interest. (B) Mean activity of all rostral neurons on trials in which the contralateral target was selected. Blue circles indicate GABAergic neurons. Black bars, median firing rate of all rostral neurons. ${ }^{* *} p=0.0004,{ }^{*} p=0.024$, multiple comparisons. Results were similar for trials in which the ipsilateral target was selected (not shown). (C) As in $\mathbf{B}$, for caudal neurons. 
445

446

447

448

449

450

451

452

453

454

455

456

457

458

459

460

461

462

463

464

465

466

467

468

469

\section{Intrinsic SC circuitry can support a rostral increase in activity for target acquisition}

Our results demonstrate that most choice-predictive neurons exhibit higher activity during movement to the target than during choice (Fig. 1) and that activity in rostrally recorded neurons increases during target acquisition (Figs. 2; 3). If choice-related activity initiates a default motor plan for subsequent acquisition of selected targets, we would expect that intrinsic SC circuitry would suffice to link choice to acquisition without input from extracollicular structures (e.g., from the substantia nigra or cerebellum, both of which are known to modulate movement-related SC activity). To test for network architectures that may link target selection and acquisition in the SC, we extended an attractor model initially developed to interrogate intrinsic SC circuits for decision making (Fig. 4)(Essig et al., 2020). Briefly, the model includes a left and a right SC, each with their own populations of excitatory $(E)$ and inhibitory $(I)$ cells. Cells are arranged linearly, with rostral cells comprising the first quarter of the population and the remaining cells classified as caudal (Fig. 4A). After external excitation representing the odorants is applied to $E$ and / cells during the time steps corresponding to the choice epoch, external input is removed and the choice is read out based on the overall activity of the caudal E cells on the following step of the model (Essig et al., 2020).

We first extended the model by identifying parameters that would sustain activity well beyond the choice epoch while also preserving choice accuracy. We accomplished this by reducing the synaptic scale of $E$ cells such that recurrent excitation occurred over a smaller area and could be maintained for longer durations. However, this change did not produce a rostrally-directed shift in activity during movement, as we observed experimentally (Figs 2; 3). Therefore, guided by previous reports of long-range inhibitory interactions between rostral and caudal populations (Meredith and Ramoa, 1998; Munoz and Istvan, 1998; Behan et al., 2002) and rostrocaudal inhibitory asymmetries in the SC (Bayguinov et al., 
2015), we introduced asymmetry to the inhibitory projections based on the rostrocaudal position of the inhibitory cells (Fig. 4A). Interestingly, when we increased the spatial influence of rostral I cells $(I-$ scale), activity emerged during choice in caudal and rostral populations (Fig. 4B), despite providing external input exclusively to caudal cells during the choice epoch.

\section{A}

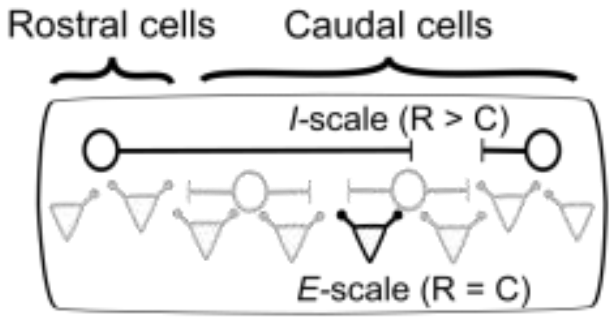

O I cells (inhibitory neurons) $\nabla E$ cells (excitatory neurons)

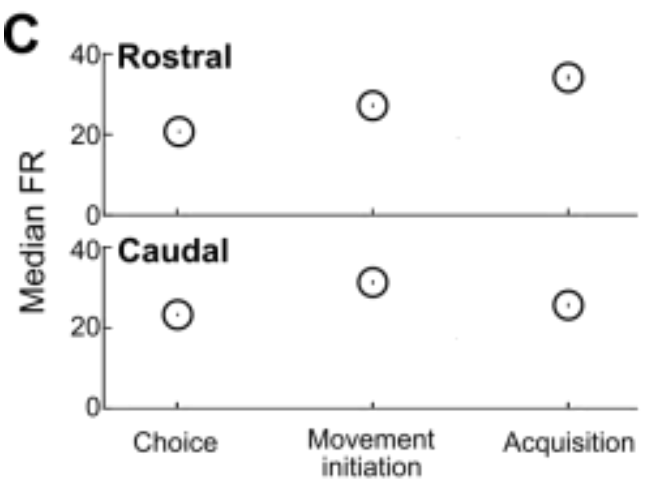

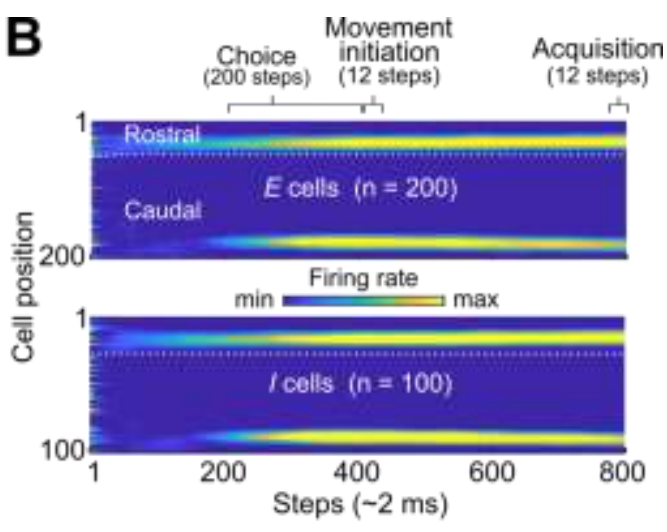

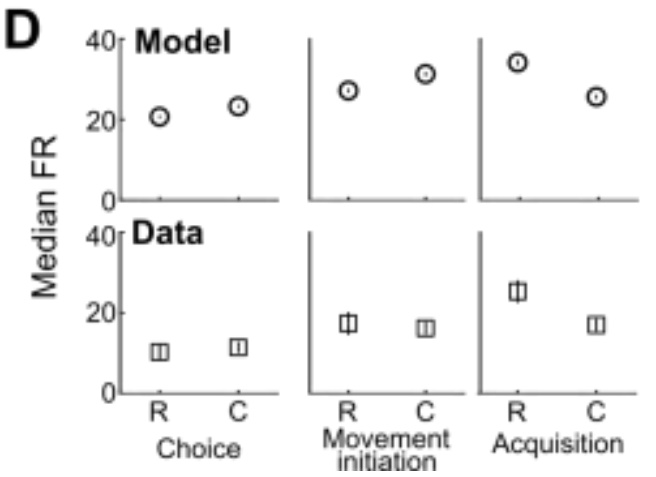

Figure 4: Attractor model captures spatiotemporal dynamics of neural activity during the task.

(A) Schematic of model. $I$ - and $E$-scale refer to range of inhibition and excitation, respectively, over which the strength of the synaptic influence decays. Rostral neurons comprise the first quarter of cells, the rest are caudal. (B) Example activity of excitatory $(E)$ cells (top) and inhibitory ( $I$ cells (bottom) on a single trial. Epochs were identified for comparison with neural activity during the task. External excitation is delivered to the most caudal $E$ and I cells only during the choice epoch. Dotted line shows division between rostral and caudal populations. (C) Median firing rates for each epoch on contralateral trials of rostral (top) and caudal (bottom) E cells averaged over 20 sessions. Error bars, +/- SEM. (D) Median rostral and caudal firing rates across epochs from the model (top) and from neural recordings (bottom). 
To determine how modeled activity evolved from target selection to acquisition, we identified model epochs that were analogous to the epochs used for our experimental data (Fig. 4B). Each step in the model is equivalent to approximately $2 \mathrm{~ms}$ of biological time; thus, we could estimate when the movement initiation and target acquisition would occur in relation to the choice epoch and match their durations to the epochs used for the experimental data (Fig. 4B). Similar to our experimental results, rostral activity increased for the acquisition epoch whereas caudal activity remained relatively stable for each epoch following the choice epoch (Fig. 4C). Figure 4D compares the activity of rostral and caudal populations for each epoch in the model (top) and for the experimental data (bottom). In general, the model was able to recapitulate the relative activity across epochs in the rostral and caudal populations, including the increase in rostral activity during target acquisition. We next examined how the model results depended on the built-in rostrocaudal inhibitory asymmetry (Fig. 4A). Since we were primarily interested in the relative difference

504 in firing rate between rostral and caudal SC populations, we directly compared the model results to the data by calculating the difference in rostral and caudal firing rates for each epoch (Fig. 5A). We then increased or decreased the rostral $l$-scale parameter, re-ran the

507 model, and focused on activity during the acquisition epoch, in which we observed the 508 greatest relative increase in rostral activity (Fig. 5A). Decreasing the rostral I-scale 509 parameter resulted in less activity accumulating rostrally during acquisition (Fig. 5B, top) whereas increasing the rostral $I$-scale parameter resulted in a pronounced increase in 511 rostral activity and a severe decrease in caudal activity (Fig. 5B, bottom). We repeated this 512 analysis for a range of values of the rostral $l$-scale parameter to reveal that our results 513 depended strongly on the rostral-caudal I-scale ratio (Fig. 5C), suggesting a possible 514 dependence of the rostrocaudal balance of activity on the relative spatial influence of rostral 515 and caudal inhibitory SC cells. 
A

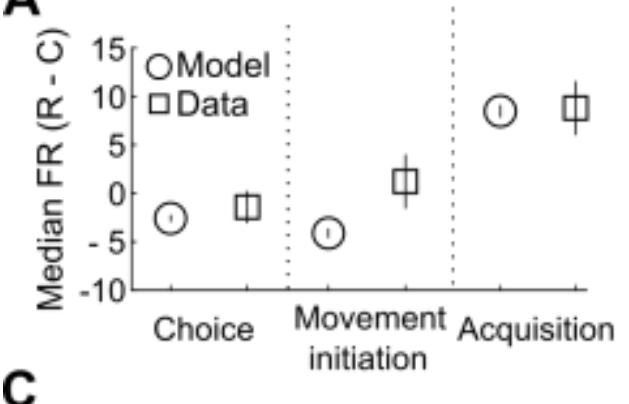

C

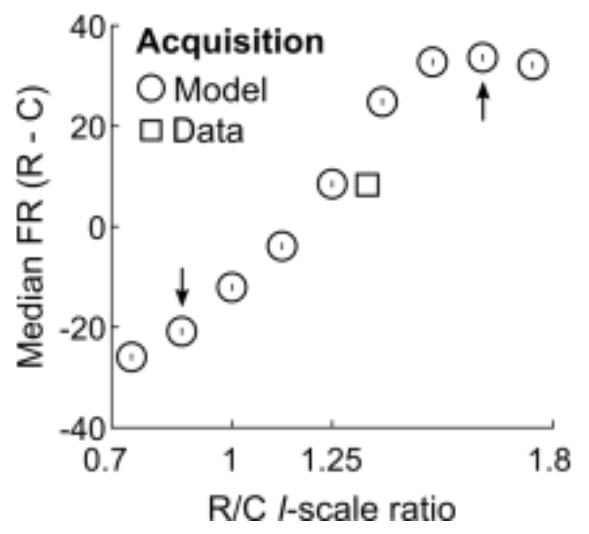

B
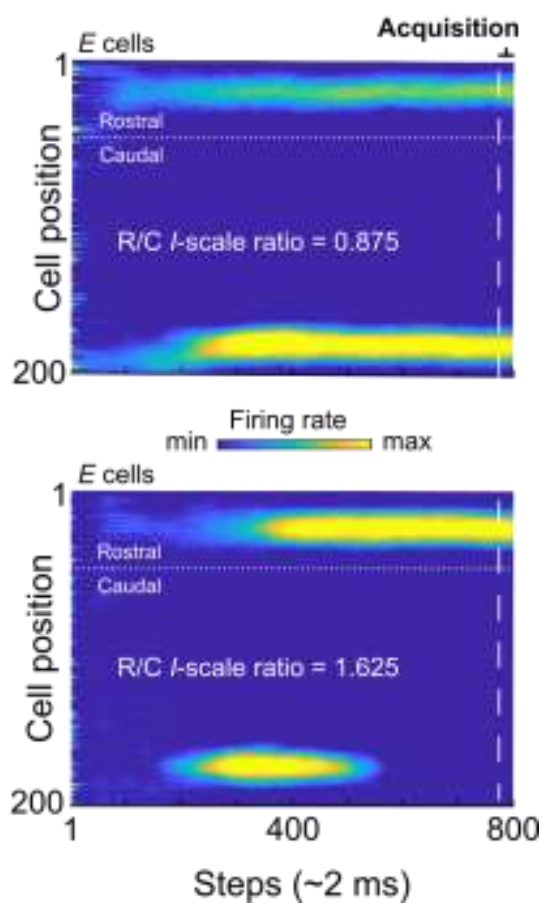

Figure 5: Rostrocaudal activity during target acquisition depends on relative spatial influence of rostral and caudal inhibitory cells.

(A) Difference between rostral and caudal (R-C) median firing rates for the model and neural recordings for each epoch. (B) Example activity of model $E$ cells for two R/C $l$-scale ratios on a single trial when caudal $l$-scale is greater than rostral $l$-scale (top) and rostral $l$ scale is far greater than caudal I-scale (bottom). Dashed lines indicate beginning of acquisition epoch. (C) Difference in rostral and caudal median E-cell firing rates during the acquisition epoch for a range of rostral/caudal $(\mathrm{R} / \mathrm{C}) \mathrm{l}$-scale ratios. Data from neural recordings are plotted as a square for comparison. Arrows indicate $l$-scale ratios used in $\mathbf{B}$.

Overall, the model demonstrates that choice-related activity and intrinsic SC circuitry alone, without external modulation, could account for target-acquisition-related activity and further supports the hypothesis that functional SC circuits underlying target selection provide a default motor plan for target acquisition. 


\section{Discussion}

538 In this study, we examined the idea that shared neural circuitry underlies the selection of targets for movement and their acquisition. We hypothesized that, since circuits in the superior colliculus (SC) mediate target selection and acquisition (Mays and Sparks, 1980;

541 Horwitz and Newsome, 1999; Glimcher, 2001; Guitton et al., 2003; Felsen and Mainen, 542 2008; Gandhi and Katnani, 2011; Duan et al., 2015; Basso and May, 2017), SC circuitry 543 links these processes by establishing a default motor plan for target acquisition initiated by 544 selection-related activity. By recording from rostral and caudal SC neurons while mice 545 selected, oriented to, and acquired targets, we found that many neurons were active for

546 these task-related behaviors, and that activity was generally higher during movement, even

547 in those neurons thought to play a causal role in target selection (Fig. 1). The balance of 548 activity shifted rostrally during movement, although not among the GABAergic population 549 that was more active later in the movement regardless of rostrocaudal position (Fig. 2), and

550 an attractor model limited to intrinsic SC circuits accounted for this rostral shift (Fig. 4).

551 Together, these results are consistent with the idea that functional circuitry for target 552 selection in the SC provides a default motor plan for subsequently acquiring the selected 553 target.

Our work builds on a wealth of studies examining how SC activity relates to target

555 selection and acquisition, which have mainly focused on gaze shifts (Sparks, 1999), 556 primarily via saccadic eye movements, in primates and cats (Gandhi and Katnani, 2011).

557 The full-body orienting movements required by our task appear to similarly engage the 558 mouse SC: Perturbation experiments predictably bias choices (Felsen and Mainen, 2008;

559 Stubblefield et al., 2013; Lintz et al., 2019; Essig et al., 2020), and we observed that activity 560 in rostral SC tended to peak later during movement (Fig. 2B), and was higher during target 561 acquisition (Figs. 3B,C; 4D), than activity in caudal SC. Data from the SC of primates and 
cats making saccades is broadly similar (Wolf et al., 2015), however some of our results differed from previous data. For example, we found that caudal SC activity was only slightly higher than rostral activity during the choice epoch and was even higher during acquisition than during choice (Fig. 3B,C). These results are inconsistent with a strict caudal-to-rostral shift in the locus of activity during movement, whereby caudal activity decreases as rostral activity increases, as has been described for gaze shifts (Guitton et al., 2003). Instead, our results show that rostral activity is more strongly modulated by target acquisition than caudal activity, driving a rostral spread of activity during movement without a concomitant decrease in caudal activity (Anderson et al., 1998). Thus, while we found that the balance of SC activity shifts rostrally during movement, consistent with previous results in primates and cats during saccades, activity in caudal SC during full-body movements in mice may differ from caudal SC activity in primates and cats during saccades. To examine this possibility further, simultaneous recordings at precise locations along the rostrocaudal axis would need to be performed.

Our study was the first to examine the activity of GABAergic SC neurons during target acquisition. We found that GABAergic neurons are active slightly later during movement than the overall population of SC neurons (Fig. 2), but that their activity during target acquisition was broadly similar to the overall population (Fig. 3B,C). These results suggest that, similar to their role during target selection (Essig et al., 2020), GABAergic neurons do not primarily provide local suppression: If they did, we would expect to observe qualitative differences between the activity of GABAergic and the overall population. Rather, they may provide longer-range inhibition during target acquisition. Consistent with the role of inhibition in our attractor model (Fig. 4) and a proposed mechanism for stopping saccades (Munoz and Istvan, 1998), GABAergic neurons may direct SC activity rostrally as movement occurs to ultimately promote movement cessation at the target. 
While our results support the idea that target acquisition is influenced by target selection via shared circuitry in the SC, they do not rule out a role for other brain regions in modulating target acquisition-related activity in the rostral SC. Indeed, the rostral SC integrates input from numerous brain regions (Benavidez et al., 2020; Doykos et al., 2020), many of which could contribute to processes related to target acquisition, including regulating movement speed and movement cessation. In particular, the cerebellar nuclei are thought to play a role in online motor control by modulating activity in movement-related circuitry, suggesting that their robust projections to the rostral SC contribute to target acquisition for SC-dependent orienting movements (Noorani and Carpenter, 2017). This input may be critical for retuning target acquisition when real-time sensorimotor remapping is required or may increase the precision of target acquisition under stable conditions. Thus, while a default motor plan for target acquisition in the SC may be formed during target selection, the circuitry is sufficiently flexible to allow for modulation by other structures. Our findings raise several questions that can be addressed by future studies. First, while our study and others have shown that activity in rostral SC correlates with target acquisition, it is unclear whether this relationship is causal. Electrical microstimulation and pharmacological inhibition of primate rostral SC during goal-directed eye movements affects the movement endpoint (Gandhi and Keller, 1999; Basso et al., 2000; Hafed et al., 2008; Goffart et al., 2012), but the effect of transiently inhibiting rostral SC activity during movement is not known. Restricting inhibition to specific cell types and during specific phases of movement (e.g., optogenetically) could elucidate the functional neural circuitry in SC necessary for target acquisition. In particular, the GABAergic SC neurons studied here comprise a heterogeneous population (Mize, 1992; Sooksawate et al., 2011) that plays multiple functional roles. Recording and perturbing specific types of GABAergic neurons, as well as other types of SC neurons such as those projecting to brainstem motor nuclei, 
612 during target acquisition would be valuable. In addition, our analyses examined how SC

613 circuitry could guide orienting movements to the target regardless of the specific effectors

614 involved, consistent with the framework that the SC performs a general role in representing

615 spatial targets (Krauzlis et al., 2004). Since the SC mediates pinnae movements (Stein and

616 Clamann, 1981), whisking (Hemelt and Keller, 2008), licking (Rossi et al., 2016) and head-

617 orientation (Corneil et al., 2002; Wilson et al., 2018), all of which undoubtedly occur during

618

619

620

621

622

623

624

625

626

627

628

629

630

631

632

633

634

635

636

637

638

639

640

641

642

643

644

645

646

647 our task, future studies can use high-resolution tracking methods (Mathis and Mathis, 2020)

while recording SC activity to examine how these body adjustments are encoded in SC

circuits as animals acquire targets. Finally, the functions of external inputs to the rostral SC

during target acquisition can be examined, towards the larger goal of understanding how

the SC integrates input from a network of brain regions to coordinate goal-directed

behavior.

\section{References}

Anderson, RW, Keller, EL, Gandhi, NJ, and Das, S. (1998). Two-dimensional saccaderelated population activity in superior colliculus in monkey. J. Neurophysiol. 80, 798-817.

Anikeeva, P, Andalman, AS, Witten, I, Warden, M, Goshen, I, Grosenick, L, Gunaydin, LA, Frank, LM, and Deisseroth, K. (2012). Optetrode: a multichannel readout for optogenetic control in freely moving mice. Nat. Neurosci. 15, 163-170.

Basso, MA, Krauzlis, RJ, and Wurtz, RH. (2000). Activation and Inactivation of Rostral Superior Colliculus Neurons During Smooth-Pursuit Eye Movements in Monkeys.

Basso, MA, and May, PJ. (2017). Circuits for Action and Cognition: A View from the Superior Colliculus. Annu. Rev. Vis. Sci. 3, 197-226.

Basso, MA, and Wurtz, RH. (1998). Modulation of neuronal activity in superior colliculus by changes in target probability. J. Neurosci. 18, 7519-7534.

Bayguinov, PO, Ghitani, N, Jackson, MB, and Basso, MA. (2015). A hard-wired priority map in the superior colliculus shaped by asymmetric inhibitory circuitry. J. Neurophysiol. 114, 662-676.

Behan, M, Steinhacker, K, Jeffrey-Borger, S, and Meredith, MA. (2002). Chemoarchitecture of GABAergic neurons in the ferret superior colliculus. J. Comp. Neurol. 452, 334-359. 
Benavidez, NL et al. (2020). The mouse cortico-tectal projectome. BioRxiv., 2020.03.24.006775.

Bergeron, A, Matsuo, S, and Guitton, D. (2003). Superior colliculus encodes distance to target, not saccade amplitude, in multi-step gaze shifts. Nat. Neurosci. 6, 404-413.

Choi, WY, and Guitton, D. (2006). Responses of Collicular Fixation Neurons to Gaze Shift Perturbations in Head-Unrestrained Monkey Reveal Gaze Feedback Control. Neuron. 50, 491-505.

Choi, WY, and Guitton, D. (2009). Firing patterns in superior colliculus of head-unrestrained monkey during normal and perturbed gaze saccades reveal short-latency feedback and a sluggish rostral shift in activity. J. Neurosci. 29, 7166-7180.

Cisek, P. (2019). Resynthesizing behavior through phylogenetic refinement. Attention, Perception, Psychophys. 81, 2265-2287.

Cisek, P, and Kalaska, JF. (2010). Neural mechanisms for interacting with a world full of action choices. Annu. Rev. Neurosci. 33, 269-298.

Cisek, P, and Pastor-Bernier, A. (2014). On the challenges and mechanisms of embodied decisions. Philos. Trans. R. Soc. B Biol. Sci. 369.

Corneil, BD, Olivier, E, and Munoz, DP. (2002). Neck muscle responses to stimulation of monkey superior colliculus. I. Topography and manipulation of stimulation parameters. J. Neurophysiol. 88, 1980-1999.

Doykos, TK, Gilmer, JI, Person, AL, and Felsen, G. (2020). Monosynaptic inputs to specific cell types of the intermediate and deep layers of the superior colliculus. J. Comp. Neurol. 528, 2254-2268.

Duan, CA, Erlich, JC, and Brody, CD. (2015). Requirement of Prefrontal and Midbrain Regions for Rapid Executive Control of Behavior in the Rat. Neuron. 86, 1491-1503.

Essig, J, Hunt, J, and Felsen, G. (2020). Inhibitory midbrain neurons mediate decision making. BioRxiv., 2020.02.25.965699.

Everling, S, Dorris, MC, Klein, RM, and Munoz, DP. (1999). Role of primate superior colliculus in preparation and execution of anti-saccades and pro-saccades. J. Neurosci. 19, 2740-2754.

Feierstein, CE, Quirk, MC, Uchida, N, Sosulski, DL, and Mainen, ZF. (2006).

Representation of Spatial Goals in Rat Orbitofrontal Cortex. Neuron. 51, 495-507.

Felsen, G, and Mainen, ZF. (2008). Neural Substrates of Sensory-Guided Locomotor Decisions in the Rat Superior Colliculus. Neuron. 60, 137-148.

Franklin, KBJ, and Paxinos, G. (2008). The mouse brain in stereotaxic coordinates, Cambridge: Academic Press. 
Gandhi, NJ, and Katnani, HA. (2011). Motor functions of the superior colliculus. Annu. Rev. Neurosci. 34, 205-231.

Gandhi, NJ, and Keller, EL. (1999). Comparison of saccades perturbed by stimulation of the rostral superior colliculus, the caudal superior colliculus, and the omnipause neuron region. J. Neurophysiol. 82, 3236-3253.

Glimcher, PW. (2001). Making choices: the neurophysiology of visual-saccadic decision making. Trends Neurosci. 24, 654-659.

Goffart, L, Hafed, ZM, and Krauzlis, RJ. (2012). Visual Fixation as Equilibrium: Evidence from Superior Colliculus Inactivation. J. Neurosci. 32, 10627-10636.

Green, DM, and Swets, JA. (1966). Signal detection theory and psychophysics, New York: Wiley.

Guitton, D, Bergeron, A, Choi, WY, and Matsuo, S. (2003). On the feedback control of orienting gaze shifts made with eye and head movements. In: Progress in Brain Research, Elsevier, 55-68.

Hafed, ZM, Goffart, L, and Krauzlis, RJ. (2008). Superior colliculus inactivation causes stable offsets in eye position during tracking. J. Neurosci. 28, 8124-8137.

Hemelt, ME, and Keller, A. (2008). Superior colliculus control of vibrissa movements. J. Neurophysiol. 100, 1245-1254.

Horwitz, GD, and Newsome, WT. (1999). Separate signals for target selection and movement specification in the superior colliculus. Science (80-. ). 284, 1158-1161.

Horwitz, GD, and Newsome, WT. (2001). Target Selection for Saccadic Eye Movements: Prelude Activity in the Superior Colliculus During a Direction-Discrimination Task. J. Neurophysiol. 86, 2543-2558.

Isa, K, Sooksawate, T, Kobayashi, K, Kobayashi, K, Redgrave, P, and Isa, T. (2020). Dissecting the tectal output channels for orienting and defense responses. ENeuro.

Krauzlis, RJ. (2003). Neuronal activity in the rostral superior colliculus related to the initiation of pursuit and saccadic eye movements. J. Neurosci. 23, 4333-4344.

Krauzlis, RJ, Liston, D, and Carello, CD. (2004). Target selection and the superior colliculus: goals, choices and hypotheses. Vision Res. 44, 1445-1451.

Lintz, MJ, Essig, J, Zylberberg, J, and Felsen, G. (2019). Spatial representations in the superior colliculus are modulated by competition among targets. Neuroscience. 408, $191-$ 203.

Marino, RA, Levy, R, Boehnke, S, White, BJ, Itti, L, and Munoz, DP. (2012). Linking visual response properties in the superior colliculus to saccade behavior. Eur. J. Neurosci. 35, 
1738-1752.

Masullo, L, Mariotti, L, Alexandre, N, Freire-Pritchett, P, Boulanger, J, and Tripodi, M. (2019). Genetically Defined Functional Modules for Spatial Orienting in the Mouse Superior Colliculus. Curr. Biol. 29, 2892-2904.e8.

Mathis, MW, and Mathis, A. (2020). Deep learning tools for the measurement of animal behavior in neuroscience. Curr. Opin. Neurobiol. 60,1-11.

Mays, LE, and Sparks, DL. (1980). Dissociation of visual and saccade-related responses in superior colliculus neurons. J. Neurophysiol. 43, 207-232.

McPeek, RM, and Keller, EL. (2002). Superior Colliculus Activity Related to Concurrent Processing of Saccade Goals in a Visual Search Task. J. Neurophysiol. 87, 1805-1815. Meredith, MA, and Ramoa, AS. (1998). Intrinsic circuitry of the superior colliculus: pharmacophysiological identification of horizontally oriented inhibitory interneurons. J. Neurophysiol. 79, 1597-1602.

Miller, GA, Galanter, E, and Pribram, KH. (1960). Plans and the structure of behavior., New York: Henry Holt and Co.

Mize, RR. (1992). The organization of GABAergic neurons in the mammalian superior colliculus. Prog. Brain Res. 90, 219-248.

Munoz, DP, and Istvan, PJ. (1998). Lateral inhibitory interactions in the intermediate layers of the monkey superior colliculus. J. Neurophysiol. 79, 1193-1209.

Munoz, DP, Pélisson, D, and Guitton, D. (1991). Movement of neural activity on the superior colliculus motor map during gaze shifts. Science (80-. ). 251, 1358-1360.

Munoz, DP, and Wurtz, RH. (1993). Fixation cells in monkey superior colliculus I. Characteristics of cell discharge. J. Neurophysiol. 70, 559-575.

Munoz, DP, and Wurtz, RH. (1995a). Saccade-related activity in monkey superior colliculus. I. Characteristics of burst and buildup cells. J. Neurophysiol. 73, 2313-2333.

Munoz, DP, and Wurtz, RH. (1995b). Saccade-related activity in monkey superior colliculus. II. Spread of activity during saccades. J. Neurophysiol. 73, 2334-2348.

Noorani, I, and Carpenter, RHS. (2017). Not moving: the fundamental but neglected motor function. Philos. Trans. R. Soc. Lond. B. Biol. Sci. 372.

Port, NL, Sommer, MA, and Wurtz, RH. (2000). Multielectrode Evidence for Spreading Activity Across the Superior Colliculus Movement Map. J. Neurophysiol. 84, 344-357.

Rossi, MA et al. (2016). A GABAergic nigrotectal pathway for coordination of drinking behavior. Nat. Neurosci. 19, 742-748.

Schmitzer-Torbert, N, Jackson, J, Henze, D, Harris, K, and Redish, AD. (2005). Quantitative 
measures of cluster quality for use in extracellular recordings. Neuroscience. 131, 1-11.

Smalianchuk, I, Jagadisan, UK, and Gandhi, NJ. (2018). Instantaneous midbrain control of saccade velocity. J. Neurosci. 38, 10156-10167.

Sooksawate, T, Isa, K, Behan, M, Yanagawa, Y, and Isa, T. (2011). Organization of GABAergic inhibition in the motor output layer of the superior colliculus. Eur. J. Neurosci. 33, 421-432.

Sparks, DL. (1999). Conceptual issues related to the role of the superior colliculus in the control of gaze. Curr. Opin. Neurobiol. 9, 698-707.

Sparks, DL, and Mays, LE. (1980). Movement fields of saccade-related burst neurons in the monkey superior colliculus. Brain Res. 190, 39-50.

Stein, BE, and Clamann, HP. (1981). Control of Pinna Movements and Sensorimotor Register in Cat Superior Colliculus. Brain. Behav. Evol. 19, 180-192.

Stein, BE, and Stanford, TR. (2008). Multisensory integration: current issues from the perspective of the single neuron. Nat. Rev. Neurosci. 9, 255-266.

Stubblefield, EA, Costabile, JD, and Felsen, G. (2013). Optogenetic investigation of the role of the superior colliculus in orienting movements. Behav. Brain Res. 255, 55-63.

Uchida, N, and Mainen, ZF. (2003). Speed and accuracy of olfactory discrimination in the rat. Nat. Neurosci. 6, 1224-1229.

Wang, L, McAlonan, K, Goldstein, S, Gerfen, CR, and Krauzlis, RJ. (2020). A causal role for mouse superior colliculus in visual perceptual decision-making. J. Neurosci. 40, 3768-3782.

Wilson, JJ, Alexandre, N, Trentin, C, and Tripodi, M. (2018). Three-Dimensional Representation of Motor Space in the Mouse Superior Colliculus. Curr. Biol. 28, 17441755.e12.

Wolf, AB, Lintz, MJ, Costabile, JD, Thompson, J a, Stubblefield, E a., and Felsen, G. (2015). An integrative role for the superior colliculus in selecting targets for movements. J. Neurophysiol. 4532, jn.00262.2015.

Wolpert, DM, and Landy, MS. (2012). Motor control is decision-making. Curr. Opin. Neurobiol. 22, 996-1003. 